\title{
Transverse Profile of the Electron Beam for the RHIC Electron Lenses
}

\author{
X. Gu, Z. Altinbas, M. Costanzo, W. Fischer, D.M. Gassner, J. Hock, \\ Y. Luo, T. Miller, Y. Tan, P. Thieberger, C. Montag, and A.I. Pikin
}

\section{Abstract}

The transverse profile of the electron beam plays a very important role in assuring the success of the electron lens beam-beam compensation, as well as its application in space charge compensation. To compensate for the beam-beam effect in the Relativistic Heavy lon Collider (RHIC) at Brookhaven National Laboratory, we recently installed and commissioned two electron lenses. In this paper, we describe, via theory and simulations using the code Parmela, the evolution of the density of the electron beam with space charge within an electron lens from the gun to the main solenoid. Our theoretical analysis shows that the change in the beam transverse density is dominated by the effects of the space charge induced longitudinal velocity reduction, not by those of transverse Coulomb collisions. We detail the transverse profile of RHIC electron-lens beam, measured via the YAG screen and pinhole detector, and also describe its profile that we assessed from the signal of the electron-backscatter detector (eBSD) via scanning the electron beam with respect to the RHIC beam. We verified, in simulations and experiments, that the distribution of the transverse electron beam is Gaussian throughout its propagation in the RHIC electron lens.

\section{Motivation}

During the $2013255 \mathrm{GeV}$ proton run of the Relativistic Heavy lon Collider, we found that the threshold of the intensity of the proton bunch in RHIC was about $2 \times 10^{11}$ [1], as was predicted by the beam-beam simulations [2]. To further increase the bunch intensity, and therefore, its luminosity via compensating for the large beam-beam tune spread from the proton-proton interactions at IP6 and IP8, we installed two electron lenses in the electron-proton interaction region IR10 [3] and commissioned them during the RHIC 2013 and 2014 runs. For long-range compensation of beam-beam tune shifts [4], electron lenses had been installed and operated in the Fermilab Tevatron collider [5, 6, 7]. They were also used for head-on beam-beam compensation [8], to remove un-captured particles in the abort gap [9], and as well as to demonstrate halo scraping with hollow electron beams [10].

In an earlier paper [2], we specified the requirements of the electron beam in the RHIC electron lens, such as its current, shape, and the distribution of the profile of the transverse beam in the interaction region. For optimum head-on beam-beam compensation, according to theory and simulation, the electron beam in the RHIC electron lens should have the same transverse distribution as does the 
proton beam, i.e., it should be a Gaussian beam [11]. According to theoretical modeling [12, p169] of the electron beam, if the effects of space charge dominate the physics of the beam, or if the Debye length is much smaller than the beam radius, then, in a state of thermal equilibrium, the beam tends to be uniform with a sharp radius. If we ignore the space charge, or if the Debye length is much greater than beam radius, then the external focusing field will dominate the physical properties of the beam physics, and the profile of the steady-state density will be Gaussian [12, p340].

The Debye length for the electron beam of the RHIC electron lens can be estimated. The electron beam is considered as having a transversely uniform density profile, with a 5.12 Ampere current and with a $4.1 \mathrm{~mm}$ radius, which corresponds approximately to a $9.7 \mathrm{~A} / \mathrm{cm}^{2}$ max current density of the Gaussian beam for the RHIC electron lens. According to Trak simulations, the initial rms transverse temperature (velocity) just after the electron gun, which is dominated by the curved cathode surface, is approximately $12.42 \mathrm{eV}$ for a $10 \mathrm{kV}$ potential difference between the anode and the cathode. The beam is compressed by a factor of $\sqrt{20}$ via a $0.3 \mathrm{~T}$ magnetic field on the cathode, and a $6 \mathrm{~T}$ field in the interaction region. The beam size then changes from $4.1 \mathrm{~mm}$ to $0.917 \mathrm{~mm}$, and the rms transverse temperature changes to $248 \mathrm{eV}$.

During propagation within a drift tube or vacuum chamber, a $10 \mathrm{keV}$ electron beam will have a Debye length [12, p165] of $0.21 \mathrm{~mm}$, which is less than the beam radius of $0.917 \mathrm{~mm}$. By comparing the Debye length with the transverse beam size, one can find that there is a large number of electrons in a Debye sphere, which indicates that single particle scattering is negligible compared to the smoothed space charge field effect. Therefore, to evaluate the effect of space charge on the profile of the electron beam for the RHIC electron lens, some detailed studies including space charge code simulation and theoretical analyses, are necessary.

The evaluation of the transverse profile in the electron lens is critical not only for the RHIC electron lens, but also for the LHC proposed hollow-beam electron lens [13] and the integrable Optics Test Accelerator in Fermilab $[14,15]$, as well as for the application of the electron lens in space charge compensation $[16,17]$.

Another important factor that may affect transverse beam profile is diocotron instability $[18,19]$ or diocotron frequency [20]. But, for the electron beam transverse profile like the transverse profile of the RHIC electron lens, the profile can be regarded as steady because the instability evolution is sufficiently slow [18]. Even for a 1 Ampere, $5 \mathrm{keV}$ energy uniform cylindrical electron beam, a rotation of 5.76 degrees from the RHIC electron lens gun to collector is not a concern. A higher magnetic field and larger vacuum tube can reduce diocotron frequency and its effect on the transverse profile.

In this paper, we detail our evaluation of the effect of space charge on the Gaussian profile of the transverse beam, first theoretically, and then with simulations. In the Parmela [21] simulations, the beam propagates through a straight magnetic field of varying intensity; this is similar to, but shorter than, the actual magnetic field of the RHIC electron lens. The first simulation of space charge effects on hollow-beam electron lens is given in [22]; it was obtained via the particle-in-cell (PIC) code, WARP [23]. 
This paper is organized hereafter as follows. In Sec. II, we give an introduction to the RHIC electron lens. In Sec. III and Sec. IV, we detail the effect of space charge on the beam profile via theory and the Parmela code simulation, respectively. In Sec. V, we discuss the profile of the simulated beam produced from a measured cathode surface. Thereafter, we detail our experimental measurements of the electron beam transverse profiles with YAG and a pinhole for different currents in Sec. VI, as well as estimating the beam profile obtained from measuring backscattered electrons. Sec. VII and Sec. VIII contain the discussion and summary.

\section{Introduction to the RHIC Electron Lens}

Fig. 1 illustrates the layout of one of the RHIC electron lenses, which has a gun for emitting the electron beam and a collector for its collection. The electron beam emerges from the electron gun in GS1 (gun solenoid \#1) and propagates into the interaction region inside the superconducting magnet via the combination of a gun-side bending solenoid (GSB), and a superconducting magnetic field. Two correctors, GSX (gun side X steer) and GSY (gun side Y steer), control the position of the electron beam. The extent of compression of the electron beam is assured by the ratio between GS1 magnetic field and the superconducting magnetic field.

There is an electron-backscatter detector (eBSD) on the gun side dedicated to aligning the electron beam with the RHIC beam. For measuring the profile of the electron beam, there are two insertion devices, viz., a YAG screen and a pinhole detector [24]. When the electron lens runs with a high-current (e.g. 1 A) electron beam, ions may accumulate in the interaction region within the extract the accumulated ions from the interaction region, as shown in Figure 1. 


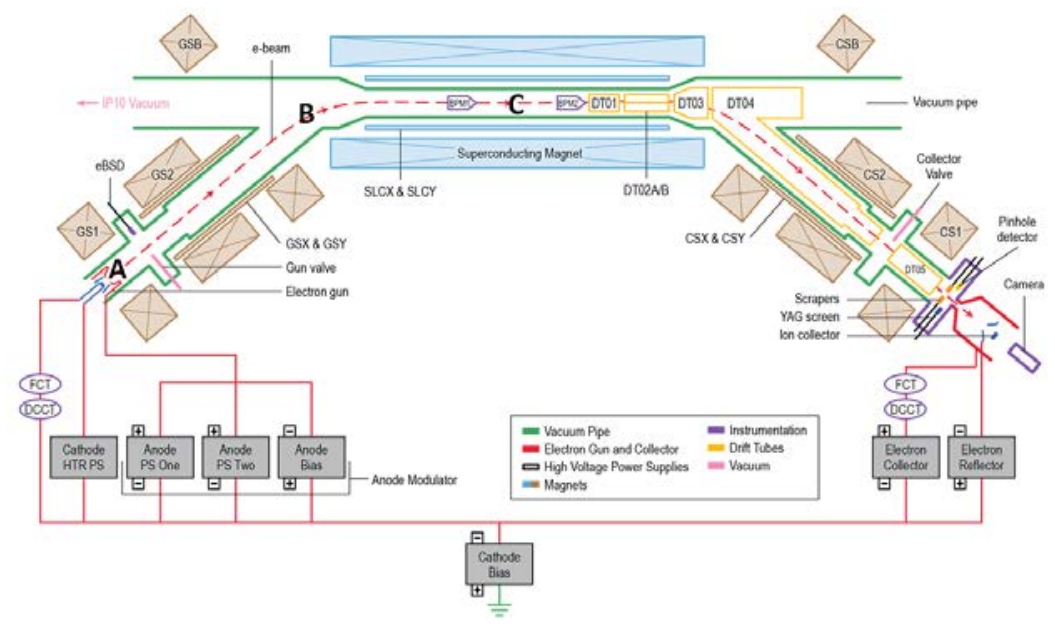

Figure 1: Schematic layout of the RHIC electron lens. The electron beam emerges from the electron gun, immersed in the magnetic field of GS1 (Gun Solenoid \#1), and propagates into the interaction region inside a superconducting magnet. The proper trajectory is accomplished via controlling fields of the GS2 (Gun Solenoid \#2) and the GSB (Gun Solenoid Bending Solenoid).

At the electron gun, the initial transverse Gaussian profile of the electron beam can be characterized by the cathode geometry [25], which was designed via the 2D program TRAK [26]. After they are manufactured, the machined cathode surfaces were projected on a screen and the surface curves were measured via a CCD camera. These measured curves then were used to simulate the beam profile, and for comparison later with the beam profile from the designed cathode. The profile of the simulated beam with its measured cathode surface predicts a Gaussian profile with a small flat top region. The results are shown in Sec. IV.

During the commissioning of the electron lenses in 2013 and 2014, the transverse profile of the electron beam was measured via both the YAG screen and pinhole scanner [24] systems. Both methods show that the electron beam still has a Gaussian transverse distribution. These measurements reveal that, as expected, the bending magnetic field does not change the beam transverse profile. We also measured the beam profile with high current $(1 \mathrm{~A})$ via the pinhole scanner. And the profile was still Gaussian, as expected. This result demonstrates that the space charge with a $1 \mathrm{~A}$ beam does not alter the beam transverse profile either.

Meanwhile, during the RHIC 2014 run, the electron backscatter detector (eBSD) for aligning the beam was commissioned and used for aligning both the electron-gold and electron- ${ }^{3} \mathrm{He}$ beams [27]. The convolution profile of electron beam and RHIC ion beam, which is the signal of the eBSD, was measured by scanning the electron beam through the ion beam. The measured convolution profile is also a Gaussian one, i.e., another demonstration of the electron beam Gaussian transverse profile. 


\section{Theory of Beam Profile and Space Charge}

The effect of space charge effect on the distribution of the electron beam density encompasses the space charge transverse effect, which is a force effect and its longitudinal effect, which is the potential-induced outcome of a reduction in velocity.

The potential created by electron beam space charge inside the beam has a transverse distribution. This causes the beam to exhibit a different longitudinal velocity in the transverse direction, which also will affect the beam transverse density distribution. There have been very few studies of the space charge induced potential or the effect of a longitudinal reduction in velocity on the beam profile.

\section{Changes in the profile of the transverse beam from the space charge transverse effect}

With the self-consistent theory of the effect of space charge on the electron beam, for the beam transverse velocities with a Maxwell-Boltzmann or thermal distribution, its stationary transverse distribution in a uniform focusing channel already has been resolved numerically [12, 28-29] for particles having the same velocity $v_{0}$, and energy $\gamma_{0} \mathrm{mc}^{2}$, in the laboratory frame, without taking into account the change in longitudinal velocity induced by the space charge potential. If space charge force effects dominate the beam physics, then the beam transverse density profile tends to be uniform with a sharp radius; if the effects of space charge force can be neglected, the beam tends to be Gaussian.

We can modify the above theory to include the effects of the longitudinal reduction in velocity occasioned by space charge potential. For those particles that are injected into a conducting drift tube or vacuum chamber (with a potential $\varphi_{\text {diff }}$ with respect to the electron beam potential) with the same kinetic energy $\left(\gamma_{0}-1\right) \mathrm{mc}^{2}=\mathrm{q} \varphi_{\text {diff }}$, the kinetic energy is then reduced by $\mathrm{q} \varphi_{\mathrm{s}}(\mathrm{r})$ because of the effect of the space charge and can be expressed as follows:

$$
\gamma(\mathrm{r}) \mathrm{mc}^{2}=\gamma_{0} \mathrm{mc}^{2}-\mathrm{q} \varphi_{\mathrm{s}}(\mathrm{r})
$$

where $\mathrm{c}$ is the speed of light, $\mathrm{m}$ is the particle mass, $q$ is the particle charge, and $\varphi_{\mathrm{s}}(\mathrm{r})$ is the beam space charge induced self-potential, which can be expressed as [12, p340]:

$$
\varphi_{\mathrm{s}}(\mathrm{r})=-\int_{0}^{r}\left[\frac{q}{\epsilon_{0} r} \cdot \int_{0}^{r} r n(r) d r\right] d r
$$

where, $n(r)$ is the beam transverse density distribution, and $\epsilon_{0}$ is the permittivity of vacuum.

Then, for the Maxwell-Boltzmann distribution of particles, equation for the transverse density profile $(5.275)$ in $[12$, p339] can be re-written after including the effect of the velocity reduction in the space charge potential:

$$
\mathrm{n}(\mathrm{r})=\mathrm{n}(0) \exp \left[-\frac{\gamma(\mathrm{r}) m v_{0}^{2}(r) \mathrm{k}_{0}^{2}(r) r^{2}}{2 k_{B} T_{\perp}}-\frac{q \varphi_{\mathrm{s}}(\mathrm{r})}{k_{B} T_{\perp} \gamma^{2}(r)}\right]
$$


where $v_{0}$ is the transverse velocity, $\mathrm{k}_{0}^{2}$ is the focus constant, $k_{B}$ is Boltzmann constant, and $T_{\perp}$ is the transverse temperature. For the uniform focusing channel of a solenoid, the constant $\mathrm{k}_{0}^{2}$ is given by $\mathrm{k}_{0}^{2}(r)=\omega_{L}^{2}(r) / v_{0}^{2}(r)[12, \mathrm{p} 312]$, and the Larmor frequency is expressed as $\omega_{L}(r)=\frac{q B}{2 \gamma(r) m}[12, \mathrm{p} 67]$. Then equation (3) can be re-written as follows:

$$
\mathrm{n}(\mathrm{r})=\mathrm{n}(0) \exp \left[-\frac{q^{2} B^{2} r^{2}}{8 k_{B} T_{\perp} \gamma(\mathrm{r}) m}-\frac{q \varphi_{\mathrm{S}}(\mathrm{r})}{k_{B} T_{\perp} \gamma^{2}(r)}\right]
$$

Equations (1), (2) and (4) are those that determine the distribution of the transverse profile of the RHIC electron lens beam. They can be solved numerically, including the space charge effect.

\section{Changes in the profile of the transverse beam due to the longitudinal effects of space charge}

With only the effect of a reduction in the space charge longitudinal potential-induced velocity, a change in beam profile can be seen for the low energy (e.g., $5 \mathrm{keV}$ ) electron beam of the RHIC electron lens.

To evaluate the effect of the velocity reduction of the space charge induced-potential on the density of the transverse beam, in Fig. 2, we show an example of a numerical calculation based on Gauss' law for a cylindrical electron beam. The space charge potential is calculated for a beam with $0.5-\mathrm{mm}$ rms beam size and $1 \mathrm{~A}$ current Gaussian electron beam which propagates in a 40-mm inner diameter drift tube. The two initial potentials between the electron beam and grounded drift tube are set to $5 \mathrm{keV}$ and $10 \mathrm{keV}$. After several iterations, the calculated space charge potentials converge as shown in Figure 2. They have their maximum absolute values at the beam center, which entails a lower velocity in the center, as well as a space charge induced energy spread. This is similar to the Boersch effect [30], in which the longitudinal temperature increases due to the effect of the space charge $[12, p 475]$.

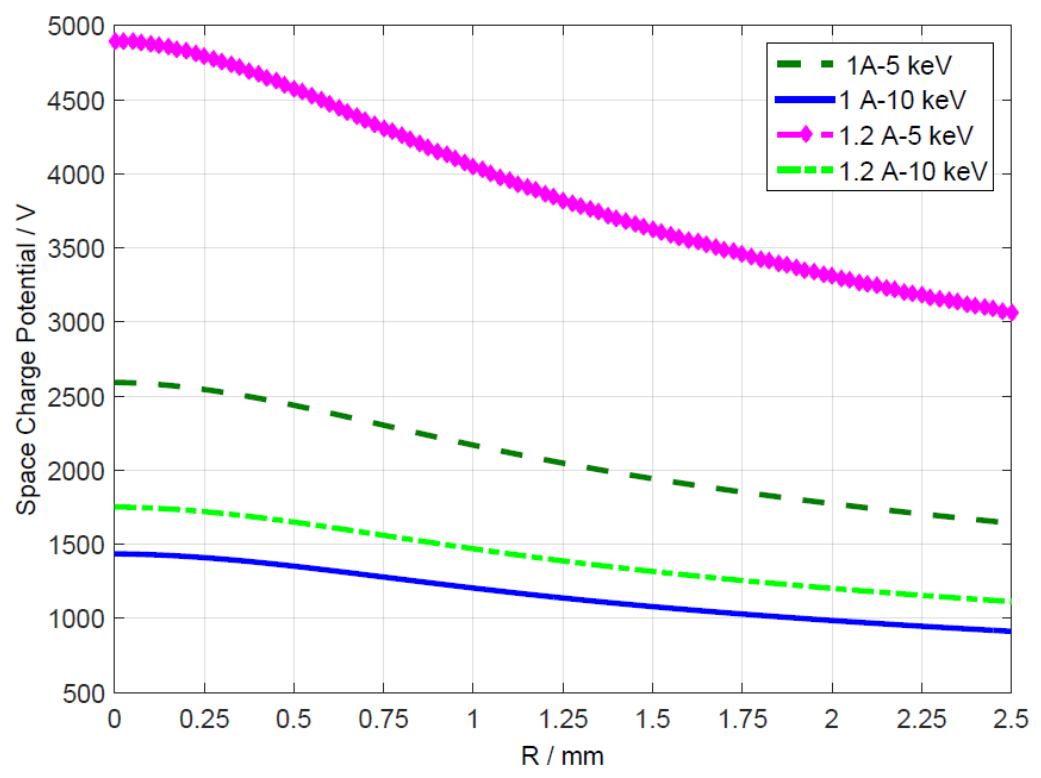


Figure 2: Space charge potentials for the $5 \mathrm{keV}$ and $10 \mathrm{keV}$ electron beams; the electron beam has $1 \mathrm{~A}$ and $1.2 \mathrm{~A}$ current, $0.5-\mathrm{mm}$ rms beam size; the internal diameter of the drift tube is $40 \mathrm{~mm}$.

Figure 2 shows that with an electron beam of $1.2 \mathrm{~A}$ current and $5 \mathrm{keV}$ energy, the space charge potential (absolute value) is very close to the beam energy. If it becomes greater than its initial energy, the electron beam will stop and become a virtual cathode, which was observed on the test bench for the RHIC electron lens [24]. With higher beam energy, this effect is reduced. We note that the electron beam current from the electron gun is determined by the anode voltage and the gun perveance; while its energy during propagation within the drift tube and vacuum chamber, other than the region earby the electron gun and collector, is determined by cathode bias voltage (Figure 1).

Figure 3 shows the electron beam transverse densities with a $1 \mathrm{~A}$ current. For a $5 \mathrm{keV}$ kineticenergy electron beam, with self-potential longitudinal velocity reduction, we found that the beam profile becomes denser in the center, and its density was lower at radius $r>0.425 \mathrm{~mm}$. With a $10 \mathrm{keV}$ beam, this effect can be neglected, as evident.

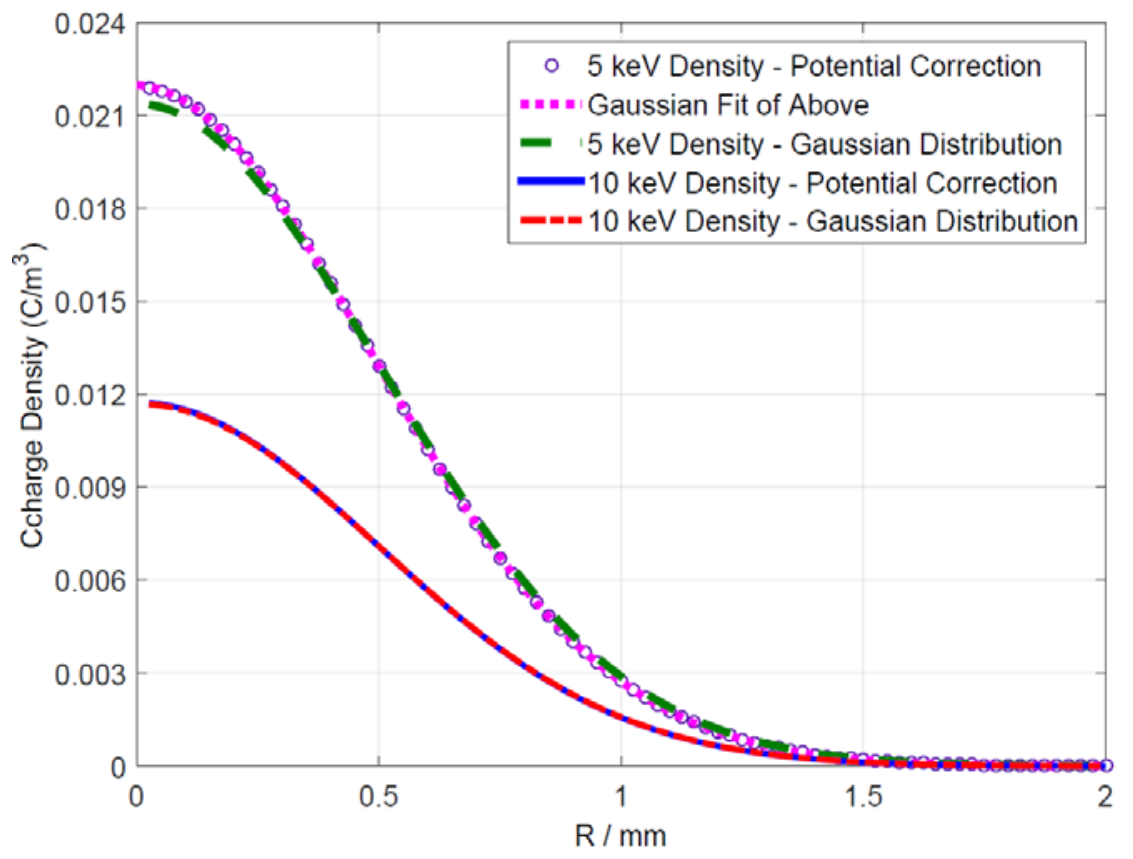

Figure 3: Beam charge density for $5 \mathrm{keV}$ and $10 \mathrm{keV}$ beams, with and without potentialinduced correction.

Fitting the profile of the $5 \mathrm{keV}$ self-potential corrected density with a Gaussian distribution, the rms beam size is reduced from $0.5 \mathrm{~mm}$ to $0.495 \mathrm{~mm}$. For the RHIC electron lens, the beam size then is not completely determined by the ratio of magnetic field because the conservation of the magnetic moment law applies only for lower energies and a constant velocity [12, p33]. From the above calculation, we find that although the effect of the space charge potential effect tends to narrow the electron beam, its transverse distribution remains close to Gaussian. 

electron beam, if the space charge effect dominates the beam physics and only Coulomb collisions are taken into account, the beam tends to be uniform with a sharp radius in the thermal equilibrium state [12, p24-25]. However, accounting only for a reduction in the space charge-induced longitudinal velocity, the beam tends to have a denser center.

However, the relaxation time toward this thermal equilibrium state is much longer than the travel time in the electron lens. The relaxation time, where the effect of the magnetic field on the relaxation toward equilibrium is not considered, can be expressed as follows [12, p474]:

$$
\tau_{e f f}=4.44 * 10^{20} * \frac{\left(k_{B} T_{e f f} / m c^{2}\right)^{1.5}}{n \ln \Delta}
$$

Where, $\ln \Delta=\ln \left[5.66 * 10^{21} * \frac{\left(k_{B} T / m c^{2}\right)^{1.5}}{n^{0.5}}\right]$ and the density $n=\frac{I}{e v \pi r^{2}} ; I$ and $r$ respectively are the beam current and radius.

Following the example in reference [12, p474] and using the same parameters as in Sec. I and reference [25], we can estimate the relaxation time and distance for a transverse, uniform RHIC electron lens beam. They are $\tau_{\text {eff }}=2.89 * 10^{-4} \mathrm{~s}$, and the distance is $1.69 * 10^{4} \mathrm{~m}$. Because the effect of a potential-induced reduction in velocity can affect the beam transverse density immediately, this means that for the RHIC electron lens, which is only a few meters long, the change in the beam transverse density is dominated by the space charge induced effect on the reduction of longitudinal velocity, not the effects of Coulomb force.

\section{Computer Simulation of the Beam Profile with Space Charge}

In the preceding section, we theoretically described how the beam shape is affected by the space charge. In this section, to reinforce that the Coulomb force effect on transverse beam profile is not the dominant effect, we evaluate the effect of space charge on the transverse profile of the RHIC electron lens beam via computer simulation. The simulated effect of space charge only includes Coulomb force effect.

We detail our evaluation of the effect of the space charge force on the Gaussian transverse distribution beam in the solenoid, using the computer simulation code Parmela [21]. This code can track particles with the space charge field that is from the particle itself, as well as from a user-defined external field. Parmela employs the routine SCHEFF [31-33] for calculating the space charge. The 2D/3D cylindrically symmetric grid algorithm can be chosen, and the mesh grid is addressed by a radial and a longitudinal bin number for 2D simulation. The space charge mesh moves at the relativistic $\gamma$ of the reference particle, and is centered on this particle. 
To treat the longitudinal distribution as a continuous beam (or DC) beam, the beam length should be much greater, e.g., 10 times longer than its diameter. In the simulation, $10^{6}$ macro-particles are used, and a 2D space charge calculation method is employed for symmetry. The external magnetic field is created via the Superfish program [21]. To avoid the reduction in the density of the beam particle due to increase in beam length, the longitudinal mesh size is set to a large value, so that the longitudinal space charge force is not calculated. Table I shows the simulation specifications.

Table 1: Specifications for Space Charge Tracking by Parmela

\begin{tabular}{c|c|c}
\hline \hline \multirow{2}{*}{$\begin{array}{c}\text { Transverse } \\
\text { Setup }\end{array}$} & Transverse Distribution & Gaussian \\
\cline { 2 - 3 } & Initial Transverse Beam Size, $\sigma$ & $1.5 \mathrm{~mm}$ \\
\cline { 2 - 3 } $\begin{array}{c}\text { Songitudinal } \\
\text { Setup }\end{array}$ & Transverse Cut-off & $4 \sigma$ \\
\cline { 2 - 3 } & Longitudinal Distribution & Uniform \\
\hline \multirow{2}{*}{$\begin{array}{c}\text { Energy and } \\
\text { Divergence }\end{array}$} & Initial Kinetic Energy & $116.8 \mathrm{~mm}$ \\
\cline { 2 - 3 } & Energy Spread & $5 \mathrm{keV}$ \\
\cline { 2 - 3 } & Initial Particle Divergence & 0 \\
\hline \multirow{2}{*}{$\begin{array}{c}\text { Simulation } \\
\text { Setup }\end{array}$} & Beam Current & $1 \mathrm{~A}$ \\
\cline { 2 - 3 } & Macro Particle number & $1 \mathrm{E} 6$ \\
\hline
\end{tabular}

231

232

233

234

235

236

237

We propagated a Gaussian beam with a round transverse profile through about $1500 \mathrm{~mm}$, inside a straight longitudinal magnetic field of varying strength without a bending field (Figure 4). Thus, we can separate the effects of this field from the effects of the space charge force. The magnetic field is very similar to the magnetic field of the RHIC electron lens, wherein the field strength varies from the source, $0.3 \mathrm{~T}$ (Tesla), towards the interaction region, at $6 \mathrm{~T}$. 


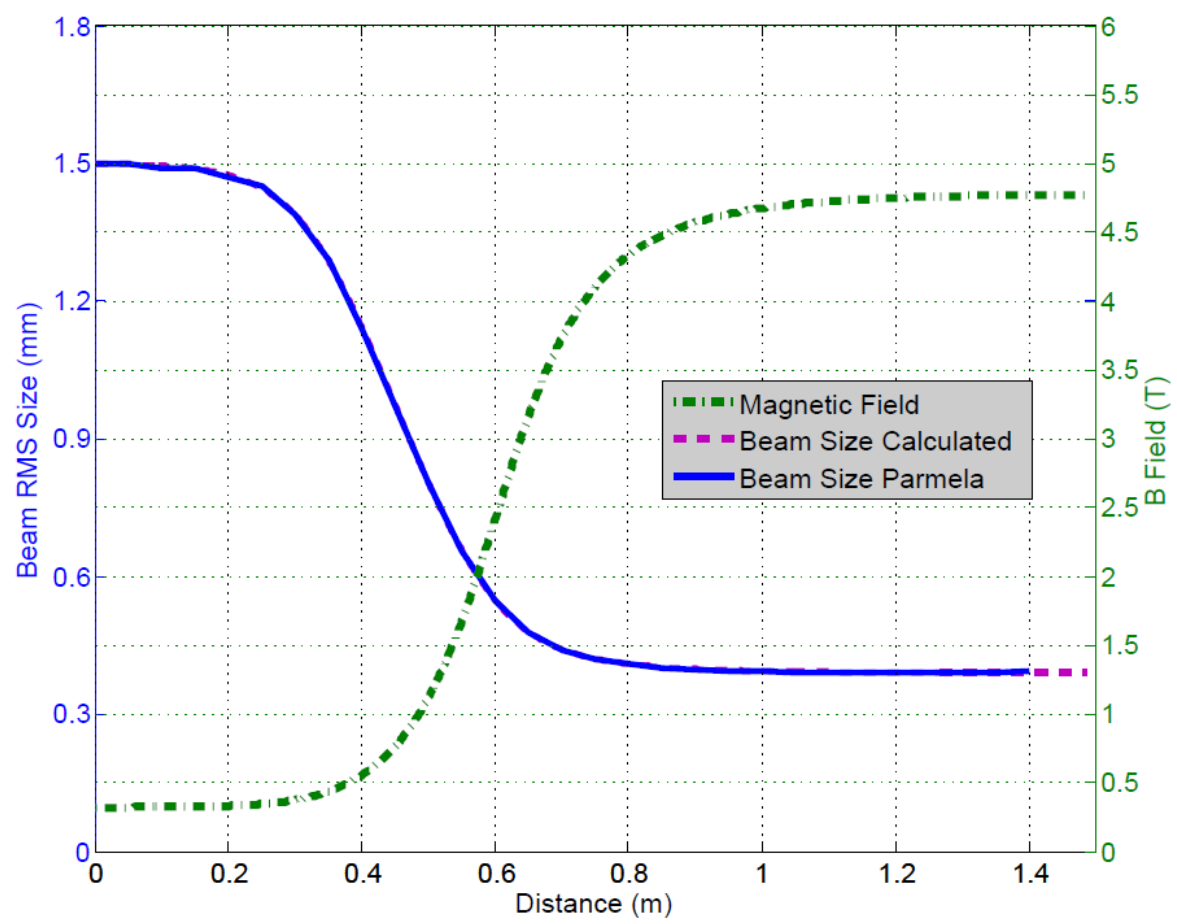

Figure 4: The straight magnetic field and the electron beam rms size.

240

241

242

243

Figure 5 shows the electron beam transverse profile at three locations, i.e., $200 \mathrm{~mm}, 500 \mathrm{~mm}$, and $1100 \mathrm{~mm}$ from the source. They correspond, respectively, to $0.33 \mathrm{~T}, 1.13 \mathrm{~T}$, and $4.73 \mathrm{~T}$ in Figure 4. The profiles are histograms of the distributions of particle number for all particles, not the particles' density distribution that is shown in Sec. III. 


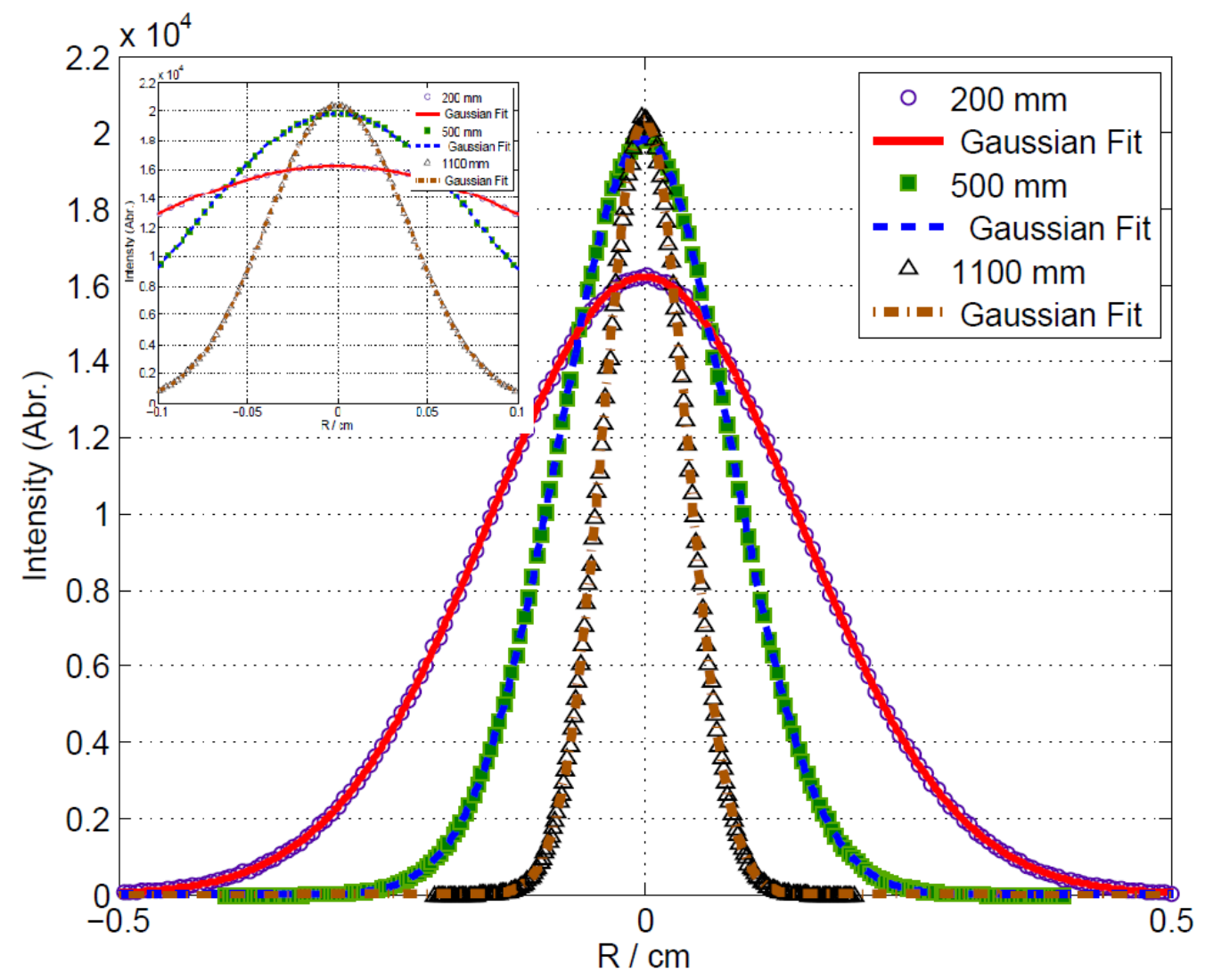

245

Figure 5: Electron density and Gaussian fit at $200 \mathrm{~mm}, 500 \mathrm{~mm}$, and $1100 \mathrm{~mm}$ of the magnetic field.

After zooming in on Figure 5, the profiles still remain Gaussian when the $1 \mathrm{~A}$ electron beam propagates from the $0.3 \mathrm{~T}$ magnetic field to the $4.7 \mathrm{~T}$ magnetic field. The theoretically calculated rms beam size and the rms size of the Parmela simulated Gaussian electron beam both shown in Figure 4 are in good agreement.

\section{Cathode and the Transverse Beam Profile}

After the cathodes were manufactured, their shapes were measured by a CCD camera via projecting them on to a screen to get two-dimensional cross-sectional profile. Figure 6 compares the designed cathode model and one example of a measured cathode profile. 


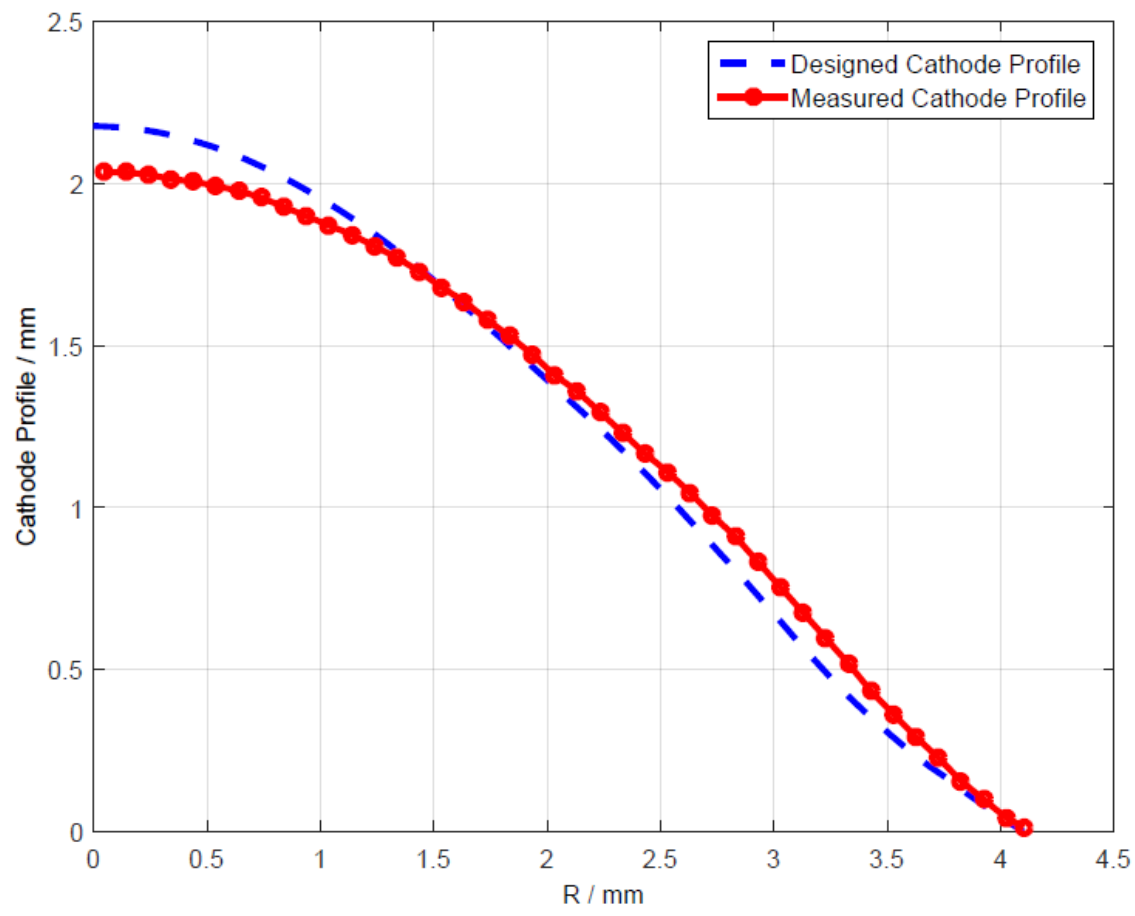

257

Figure 6: The designed and a measured cathode profiles.

There are some discrepancies between two: The machined cathode is flatter in the center than the designed one. This will result in a flat top at the center of the electron beam profile, which can be predicted by TRAK simulations based on the measured geometry. Figure 7 compares the simulated beam profiles and Gaussian fits of the designed and measured cathodes.

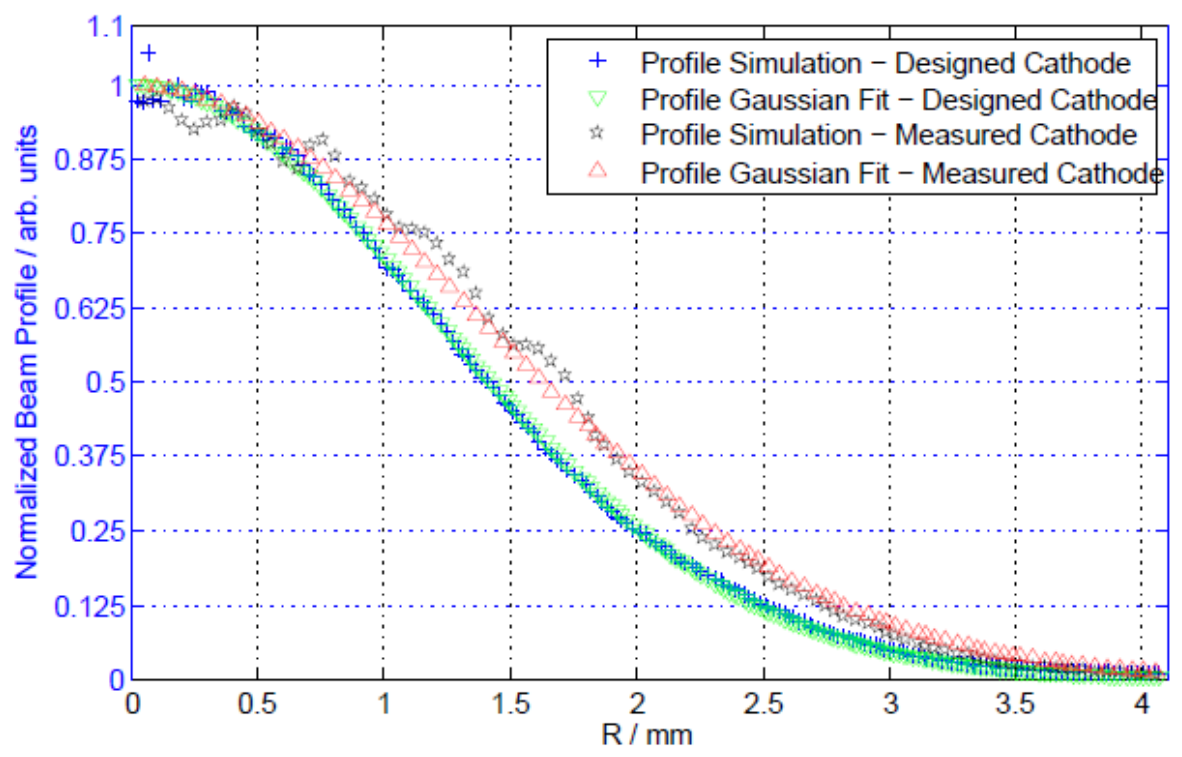


From Figure 7, we see that the Gaussian fit and the simulation of the measured cathode beam profiles agree well with each other. Moreover, there is some flattop in the simulated profile, as was confirmed by the experimentally measured profile of the beam described in the next section.

\section{Beam Profile Measurements}

\section{Beam profile measurements via YAG screen and pinhole detector}

After the 2013 RHIC run, the electron beam was propagated through the electron-lens (the layout is shown in Figure 1). Most of the system was fully tested, except for the superconducting magnet that was replaced with a 3 T superconducting magnet (the RHIC EBIS spare solenoid).

During the 2013 commissioning of the electron lens, to verify both the effect of space charge and the bending magnetic field on beam profile, the profile was measured via a pinhole detector with a $1050 \mathrm{~mA}$ beam current. The beam profile at $134 \mathrm{~mA}$ current was also measured via a YAG crystal screen to confirm agreement with the findings from the pinhole scan.
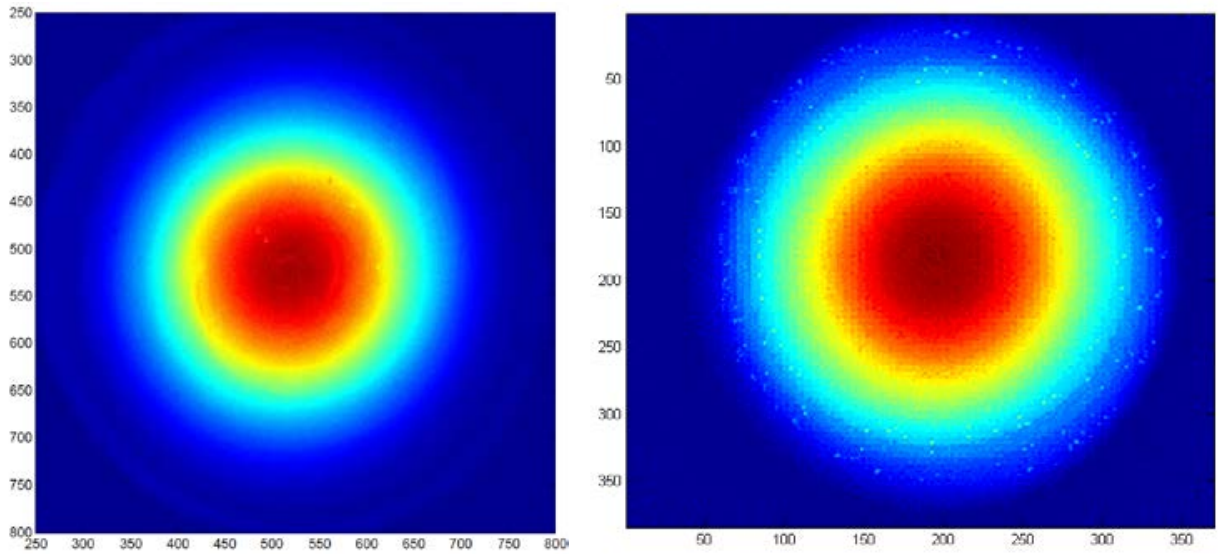

Figure 8: Beam images taken from a YAG crystal (left), and a pinhole detector (right). The pinhole image is an interpolation from $1050 \mathrm{~mA}$ beam, and 80x80-point pinhole data.

The left image in Figure 8 is the $134 \mathrm{~mA}$ beam profile measured on the YAG crystal; it qualitatively shows that the electron beam is still round. The right image is the $1050 \mathrm{~mA}$ beam profile, measured using the pinhole scanning system, and interpolated from a scan of $80 \times 80$ data points. From these images we obtained the beam transverse density distribution. 


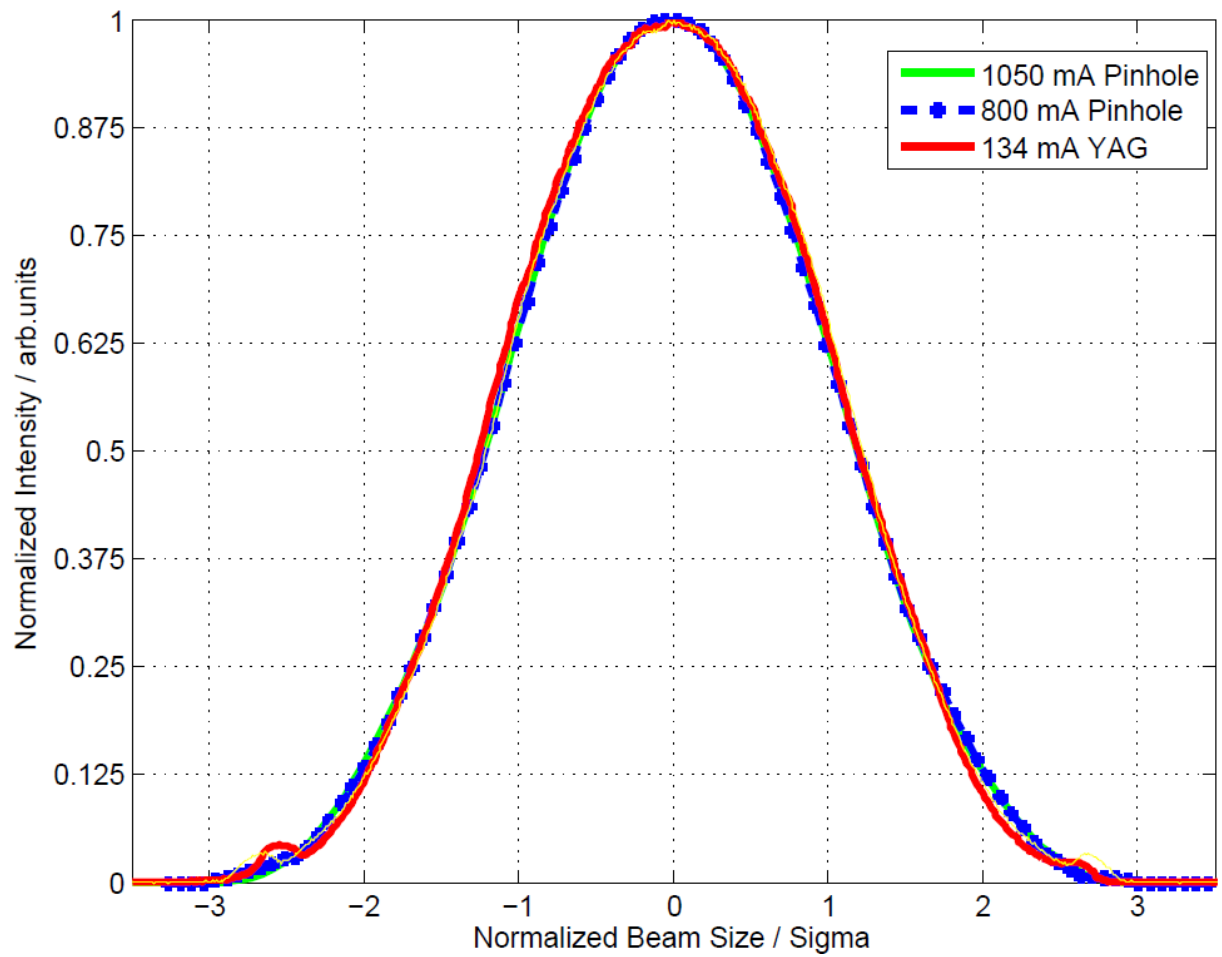

Figure 9: Beam profile from pinhole with high current, and from YAG screen with low current. The green curve is underneath the blue and red curves.

Figure 9 shows the measurements of the beam profile taken by a pinhole detector for an 800 $\mathrm{mA}$ and $1050 \mathrm{~mA}$ electron beam. They overlap very well. The profile from the YAG screen with the 134 $\mathrm{mA}$ beam current is also shown in this figure. The profiles obtained via YAG screen and pinhole detector agree well with each other.

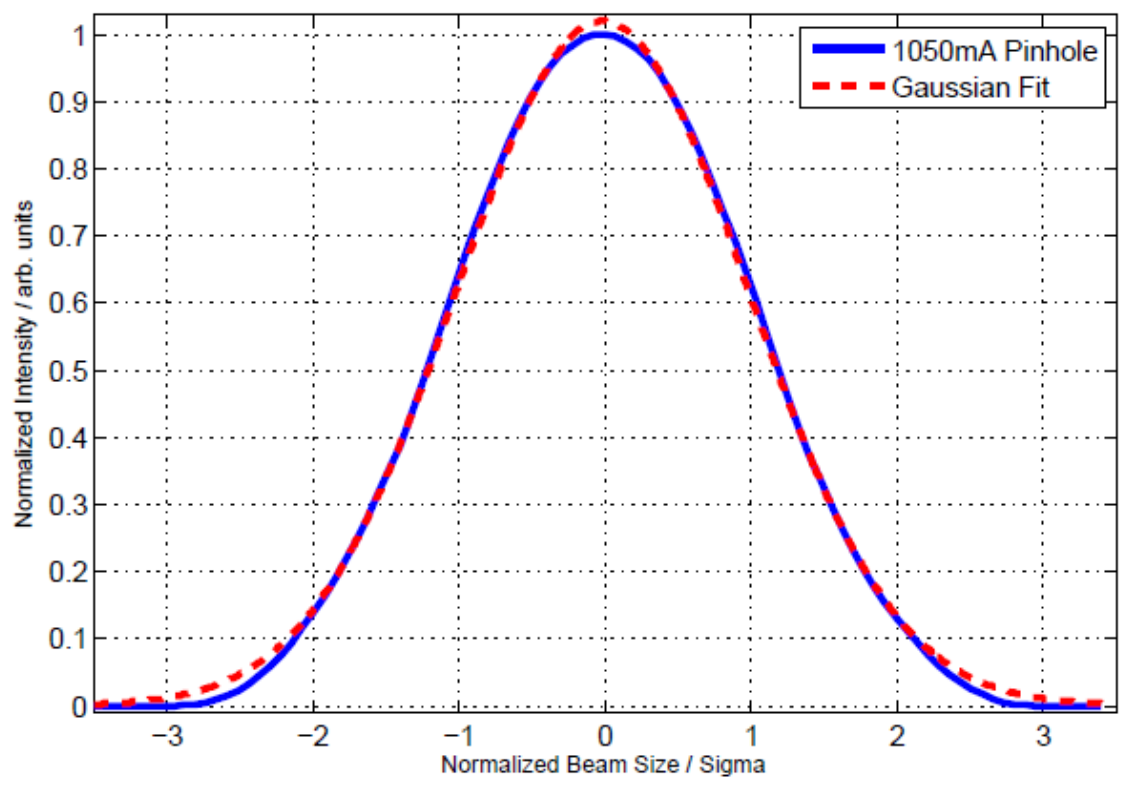


Figure 10 shows the beam profile for $1050 \mathrm{~mA}$ that was obtained from the pinhole detector together with Gaussian fit. The distribution of the Gaussian-fitted beam profile and the profile measured on the pinhole detector agree well, except in the top region of the profile, where the measured profile becomes somewhat flattened. This disparity is suspected to be caused mainly by the deviation between the model and the actual cathode surface, an interpretation that is confirmed by the comparison between the design model and the cathode geometry (or profile) measurement in Figure 7.

Another suspected reason that causes the disparity in top region of Figure 10 could be the space charge transverse velocity reduction effect (Sec. III.2). This is because both pinhole scan and YAG screen measure current density, which is the product of charge density and velocity, and the velocity distribution is not radially uniform due to this space-charge depression of the potential effect. After following the same transverse velocity reduction effect calculation in Section III.2, the variations in beam velocity with radius at YAG screen and pinhole position are about $0.16 \%, 1.09 \%$ and $1.55 \%$ for $134 \mathrm{~mA}$, $800 \mathrm{~mA}$ and $1050 \mathrm{~mA}$, respectively, with $5 \mathrm{keV}$ initial beam energy.

This $1.55 \%$ velocity variation could be another reason for the disparity in the top region of the profile in Figure 10, but this couldn't explain the region on either side of the profile in Figure 10. Furthermore, there is no visible difference between $1.09 \%$ and $1.55 \%$ velocity variation effect on beam profile for the pinhole scan profiles in Figure 9. More systemic and detailed studies about this effect could be done in the future with large energy variation and different beam current.

From these measurements of the beam profile, we verified that the beam is still a Gaussian distribution at the locations of the pinhole and YAG locations in Figure 1, after passing through a $3 \mathrm{~T}$ superconducting magnetic field and the two bending magnetic field regions (gun side and collector side), as predicted by the simulation.

\section{Indirect measurement of the electron beam profile via the RHIC ion beam}

Although the above beam profiles demonstrated the Gaussian transverse beam profile for the RHIC electron lens, they were not measured directly at the interaction region.

During the RHIC 2014 run, the electron-backscatter detector (eBSD) was commissioned and became functional in beam-beam alignment [27]. The eBSD uses the backscattered electrons, coming from the electron beam interaction with the relativistic ions or protons, as the signal for measuring, optimizing, and maintaining the beam alignment.

The intensity profile of the eBSD signal can be obtained by scanning one beam with respect to another. Figure 11 shows the scans of the vertical and horizontal separation of eBSD intensity profile via steering the $5 \mathrm{keV}$ electron beam with respect to the $100 \mathrm{GeV} /$ nucleon gold beam. 


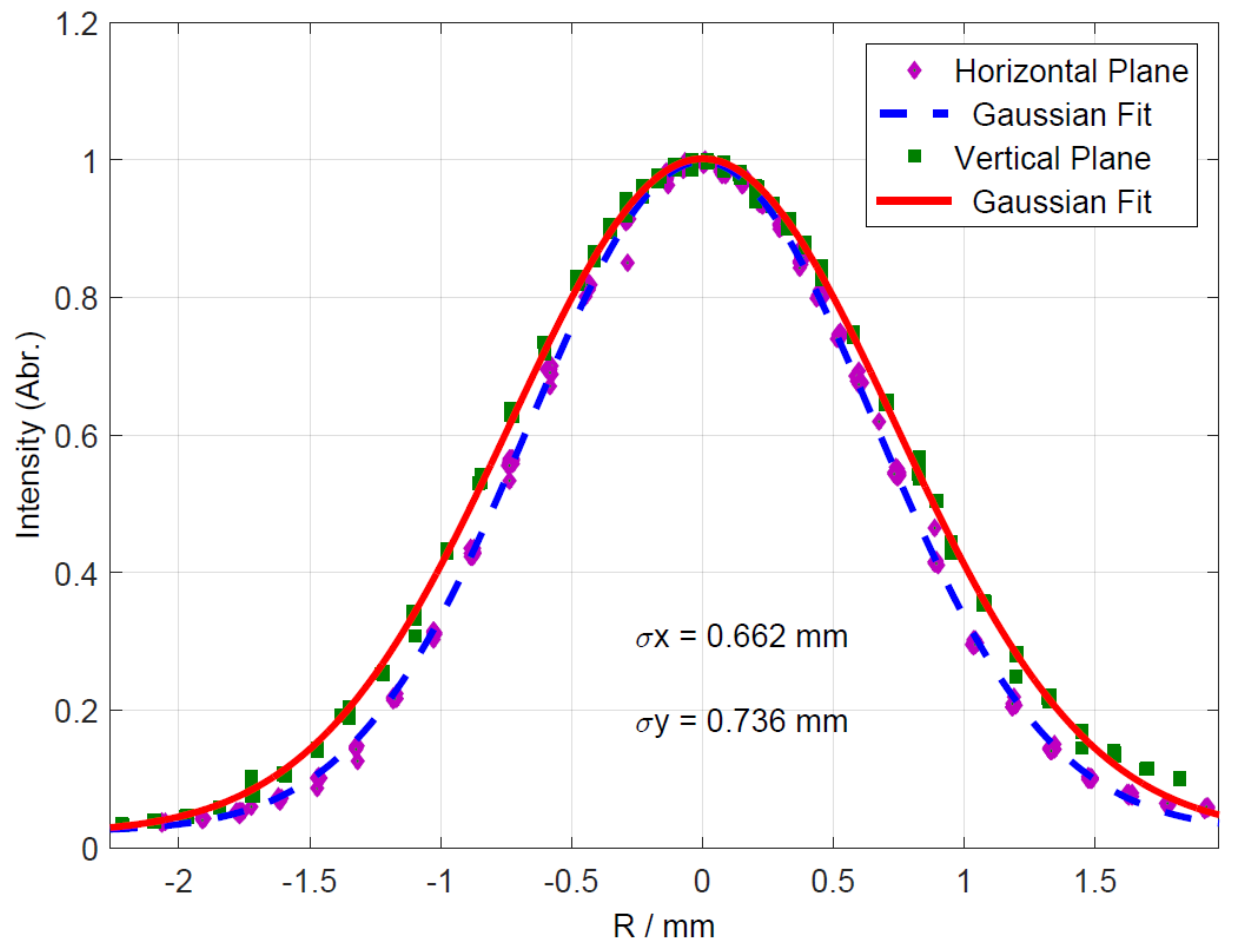

Figure 11: eBSD signals from scans of the transverse separation between the electron and gold beams.

Figure 11 shows that the eBSD signals in both the vertical and horizontal planes agree very well with a Gaussian distribution. The intensity profile of the eBSD signal is the convolution product of the profiles of the electron beam and the ion beam.

It was proved [34] that when a Gaussian beam convolutes with another Gaussian beam, their product is also Gaussian. Since the RHIC ion beam has a Gaussian distribution, and the product (the eBSD signal) of the electron beam and the ion beam has a Gaussian distribution, this is a further indication that the electron beam is also close to Gaussian.

\section{Discussion}

According to theory [35] and simulations of the propagation of single particles along the magnetic and electrostatic fields, the RHIC electron lens should avoid using transverse electrostatic fields for controlling the position of the transverse electron beam position so to ensure the undistorted shape of the electron beam [36]. Furthermore, the drift tubes in the electron lens could be used, but with care. If the electron beam is far away from the axis of the drift tube, the transverse component of the drift tube electrostatic field combined with the magnetic field can affect the beam shape.

For the RHIC electron lens, no drift tubes are used before the beam interaction region (Region C in Figure 1) to avoid a significant change in its shape via the transverse component of longitudinal drift 
tube. But after the interaction region, there is a split drift tube (DT02A/B) [37] that has a transverse electrostatic field. It can be used for clearing accumulated electrons in the magnetic bottle that forms when the superconducting magnetic field is below 2.7 T. Furthermore, some drift tubes (DT03, DT04, and DT05) are located in the exit arm. They could have some longitudinal electrostatic fields that can control the beam velocity without affecting the shape of the interacting beams. The DT01 will be used for measuring the electron beam current and pulse shape without applying any electrostatic field on it.

Although from the Parmela space charge simulations there is no apparent distortion for a $1 \mathrm{~A}$, $1.5 \mathrm{~mm}$ rms sized electron beam propagated within a magnetic field, which is similar to the electron lens, the space charge simulations were carried out with a straight magnetic field. The combination of a bending magnetic field with the space charge is not included.

Furthermore, the effect of a space charge potential-induced reduction in longitudinal velocity, which is very important for transverse beam profile, should also be included in simulating the space charge profile of the low energy electron beam (several keV). Diocotron frequency and Larmor motion effect on transverse beam profile could also be included. But neither the Parmela Code nor the routine SCHEFF includes these effects during simulation [38]. To verify the results of the Parmela simulation as well as including more space charge effect, simulations with other space charge codes, such as the PIC code Warp, could be done in the future.

Although the beam profiles, found to be Gaussian [39], were only measured at $134 \mathrm{~mA}$ (with YAG) and $1050 \mathrm{~mA}$ (with pinhole). A previous measurement of the beam profile at $664 \mathrm{~mA}$, made with the YAG crystal during the 2012 commission of the electron lens test bench, also showed a Gaussian distribution as predicted in the simulation [40].

\section{Summary}

Our theoretical study shows that the relaxation distance to the thermal equilibrium for the RHIC electron lens beam is thousands of meters. Thus, the change in the transverse beam density is dominated by the effect of the space charge induced reduction in velocity. Due to this effect, one can observe in calculating a small reduction in beam size for electron beams of $1 \mathrm{~A}$ current and $5 \mathrm{keV}$ energy. Nevertheless, the beam profile still fits well to Gaussian distribution. A higher energy of the electron beam can reduce the effect of space charge induced velocity reduction, as shown in Figure 3.

We also demonstrated with Parmela simulations, experiments on a test bench, and the experiments during 2013 and 2014 commissioning of the RHIC electron lens, that the electron beam can be fitted well to Gaussian distribution for currents up to the design value of $1 \mathrm{~A}$.

\section{Acknowledgments}


The authors would like to acknowledge the help of Jorg Kewisch and Gang Wang. We also appreciate valuable discussions with the Fermi National Accelerator Laboratory Tevatron Electron Lens (FNAL TEL) staff, in particular V. Shiltsev, A. Valishev, and G. Stancari.

The work is supported by Brookhaven Science Associates, LLC under Contract No. DE-AC02$98 \mathrm{CH} 10886$ with the U.S. Department of Energy.

\section{X. $\quad$ References}

[1] V.H. Ranjbar, V. Schoefer, L. Ahrens, et al., IPAC2013, Shanghai, China, May 12-17, 2013, pp. 15441546 (2013).

[2] Y. Luo, W. Fischer, N.P. Abreu, et al.,Phys. Rev. ST Accel. Beams 15, 051004 (2012).

[3] W. Fischer, et al., Proceedings of the ICFA Mini-Workshop on Beam-Beam Effects in Hadron Colliders (BB3013), CERN (2013).

[4] V. Shiltsev, Y. Alexahin, K. Bishofberger, et al., Physical Review Letters. 99 (24) (2007), 244801.

[5] V. Shiltsev, Y. Alexahin, K. Bishofberger, et al., Phys. Rev. ST Accel. Beams. 11 (10) (2008), 103501.

[6] V. Shiltsev, Y. Alexahin, K. Bishofberger, et al., Phys. Rev. ST Accel. Beams. 2 (7) (1999), 071001.

[7] V. Shiltsev, Y. Alexahin, K. Bishofberger, et al., New Journal of Physics 10 (4) (2008), 043042.

[8] G. Stancari, A. Valishev, in Proceedings of the ICFA Workshop on Beam-Beam Effects in Hadron Colliders (BB2013), Geneva, Switzerland, March 2013, Report No. FERMILAB-CONF-13-046-APC.

[9] X. Zhang, K. Bishofberger, V. Kamerdzhiev, et al., Phys. Rev. ST Accel. Beams 11, 051002 (2008).

[10] G. Stancari, A. Valishev, G. Annala, et al., Phys. Rev. Lett. 107 (2011), 084802.

[11] V. Shiltsev, Y Alexahin, K Bishofberger et al., New Journ.Phys. 10 (2008), 043042.

[12] M. Reiser, Theory and Design of Charged Particle Beams, Wiley-VCH Verlag GmbH \& Co. KGaA, 2008, P 33, 169, 312, 339-342, 474-475.

[13] G. Stancari, V. Previtali, and A. Valishev, et al., arXiv:1405.2033v3, FERMILAB-TM-2572-APC.

[14] G. Stancari, arXiv:1409.3615, http://arxiv.org/pdf/1409.3615v1.pdf

[15] G. Stancari, K. Carlson, M. W. McGee, et al., IPAC2015, Richmond, USA, May 4-8, 2015, MOBC3, https://jacowfs.jlab.org/conf/y15/ipac15/prepress/MOBC3.PDF

[16] V. Shiltsev, http://arxiv.org/abs/1409.1936

[17] A.V. Burov, G.W. Foster, V.D. Shiltsev, FERMILAB-TM-2125, http://Iss.fnal.gov/archive/2000/tm/TM-2125.pdf 
[18] Roy W. Gould, Phys. Phys. Plasmas, Vol. 2, No. 6, June 1995, P2151.

411 [19] R. H. Levy, Phys. Fluids 8, 1288 (1965); http://dx.doi.org/10.1063/1.1761400.

412 [20] F. Anderegg, Physics with Trapped Charged Particles, Imperial College Press (M. Knoop, N. Madsen, 413 R.C. Thompson, editors), p195-218, (2014). Chapter "Waves in Non-Neutral Plasmas",

414 http://sdpha2.ucsd.edu/pdf files/Anderegg waves leshouches 2012.pdf

415 [21] http://laacg.lanl.gov/laacg/services/serv codes.phtml

416 [22] G. Stancari, S. Redaelli, V. Moens, Proceedings of International Particle Accelerator Conference 417 (IPAC2014), Dresden, Germany, P 451-453.

418 [23] J.-L.Vay, D. P. Grote, R. H. Cohen, et al., Comput.Sci. Disc. 5, 014019 (2012).

419 [24] X. Gu, F.Z. Altinbas, E. Beebe, et al., Nucl. Instrum. Meth. Phys. Res. A743, pp. 56-67 (2014).

420 [25] A.I. Pikin, J.G. Alessi, M. Anerella, et al., Proc. 2011 Particle Accelerator Conference, New York, NY, 421 March 28-April 1, 2011, pp. 2309-2311 (2011).

422 [26] http://www.fieldp.com/

423 [27] P. Thieberger, F. Z. Altinbas, C. Carlson, et al.. Proceedings of the International Beam 424 Instrumentation Conference (IBIC) 2014.

425 [28] M. Reiser, N. Brown, Phys. Rev. Letters, 71 (1993) P 2911.

426 [29] J. D. Lawson, The Physics of Charged-Particle Beam, CLARENDON Press, Oxford, 1988, $2^{\text {nd }}$ ed., Chap. $427 \quad 4.6$.

428 [30] H. Boersch, Z. Phys. 139, 115 (1954).

429 [31] R. W. Garnett, T. P. Wangler, Proceedings of Particle Accelerator Conference (PAC1991), San 430 Francisco, California, USA, May 6-9,1991, P 330.

431 [32] E. Tanke, S. Valero, A.M. Lombardi, Proceedings of Linear Accelerator Conference 2002, Gyeongju, 432 Korea, P 656.

433 [33] P. Lapostolle, A.M. Lombardi, E. Tanke, et al, Nucl. Instrum. Meth. Phys. Res. A 379, pp. 21-40 434 (1996).

435 [34] P.A. Bromiley, Tina Memo No. 2003-003, http://www.tina-vision.net/docs/memos/2003-003.pdf

436 [35] F. F. Chen, Introduction to Plasma physics, Plenum Press, New York, 1974.

437 [36] H. Kreckel, H. Bruhns, K. A. Miller, et al., Review of Scientific Instruments, 81, 063304 (2010).

438 [37] X. Gu, A. Pikin, P. Thieberger, et al., Proc. 3rd International Particle Accelerator Conference 439 (IPAC2012), New Orleans, LA, May 20-25, 2012 pp. 4038-4040 (2012). 
440 [38] X. Pang, Los Alamos National Laboratory, Private Communication.

441 [39] T. Miller, M. Costanzo, W. Fischer, et al., "Beam Profile Measurements in the RHIC Electron Lens 442 using a Pinhole Detector and YAG Screen”, MOPF08, proc. IBIC 2014, Monterey, CA, Sept. 2014.

443 [40] A.I. Pikin, BNL-104383-2014-IR (C-A/AP/507), https://www.bnl.gov/isd/documents/85181.pdf 